\title{
Injectable Nanosponge-Loaded Pluronic FI 27 Hydrogel for Pore-Forming Toxin Neutralization
}

\author{
Shuaijun Zou ${ }^{1, *}$ \\ Qian $\mathrm{He}^{2, *}$ \\ Qianqian Wang ${ }^{l, *}$ \\ Bo Wang' \\ Guoyan Liu' \\ Fuhai Zhang' \\ Xi Cheng ${ }^{3}$ \\ Beilei Wang' \\ Liming Zhang (D) \\ 'Department of Marine Biomedicine and \\ Polar Medicine, Naval Special Medical \\ Center, Naval Medical University, \\ Shanghai, 200433, People's Republic of \\ China; ${ }^{2}$ The Third Affiliated Hospital of \\ Naval Medical University, Shanghai, \\ 200433, People's Republic of China; ${ }^{3}$ The \\ First Affiliated Hospital of Naval Medical \\ University, Shanghai, 200433, People's \\ Republic of China \\ *These authors contributed equally to \\ this work
}

Purpose: Pore-forming toxins (PFTs) perform important functions during bacterial infections. Among various virulence-targeting therapies, nanosponges (NSs) have excellent neutralization effects on multiple PFTs. To enhance treatment efficacy, NSs tend to be incorporated into other biomaterials, such as hydrogels.

Methods: In the present work, red blood cell (RBC) vesicles were harvested to wrap polymer nanoparticles, leading to the formation of NSs, and the optimal Pluronic F127 hydrogel concentration was determined for gelation. Then, a novel detoxification system was constructed by incorporating NSs into an optimized Pluronic F127 hydrogel (NS-pGel). Next, the system was characterized by rheological and sustained release behavior as well as micromorphology. Then, the in vitro neutralization effect of NS-pGel on various PFTs was examined by a hemolysis protocol. Finally, therapeutic and prophylactic detoxification efficiency was evaluated in a mouse subcutaneous infection model in vivo.

Results: A thermosensitive, injectable detoxification system was successfully constructed by loading NSs into a 30\% Pluronic F127 hydrogel. Characterization results demonstrated that the NS-pGel hybrid system sustained an ideal fluidity and viscosity at lower temperatures but exhibited a quick sol-gel transition capacity near body temperature. In addition, this hybrid system had a sustained release behavior accompanied by good biocompatibility and biodegradability. Finally, the NS-pGel system showed neutralization effects similar to those of NSs both in vitro and in vivo, indicating a good preservation of NS functionality.

Conclusion: In conclusion, we constructed a novel temperature-sensitive detoxification system with good biocompatibility and biodegradability, which may be applied to the clinical treatment of PFT-induced local lesions and infections.

Keywords: nanosponges, pore-forming toxins, detoxification, thermosensitive hydrogel, Pluronic F127

\section{Introduction}

Antibiotic-resistant bacterial infections have become a global health crisis due to high morbidity and mortality. ${ }^{1,2}$ In 2019, the World Health Organization (WHO) estimated that globally, at least 700,000 patients die from drug-resistant infections annually. Without any action, the figure might soar to 10 million by $2050 .^{3}$ Thus, there is an urgent need to actively develop new strategies against antibiotic-resistant bacteria.

Virulence factor-targeting therapies have become alternative treatments for antibiotic-resistant infections, ${ }^{4-6}$ among which biomimetic red blood cell (RBC) membrane-derived "nanosponges (NSs)" provide a novel and promising opportunity. ${ }^{7-10}$ In $2013, \mathrm{Hu}$ and colleagues ${ }^{10}$ reported for the first time that NSs
Correspondence: Beilei Wang; Liming

Zhang Center, Naval Medical University

Shanghai, 200433, People's Republic of China

Tel +862I-8I87II $29 ;+862 I-8|87| I \mid 28$

Email lilly_wang@I26.com;

Imzhang@smmu.edu.cn 


\section{Graphical Abstract}

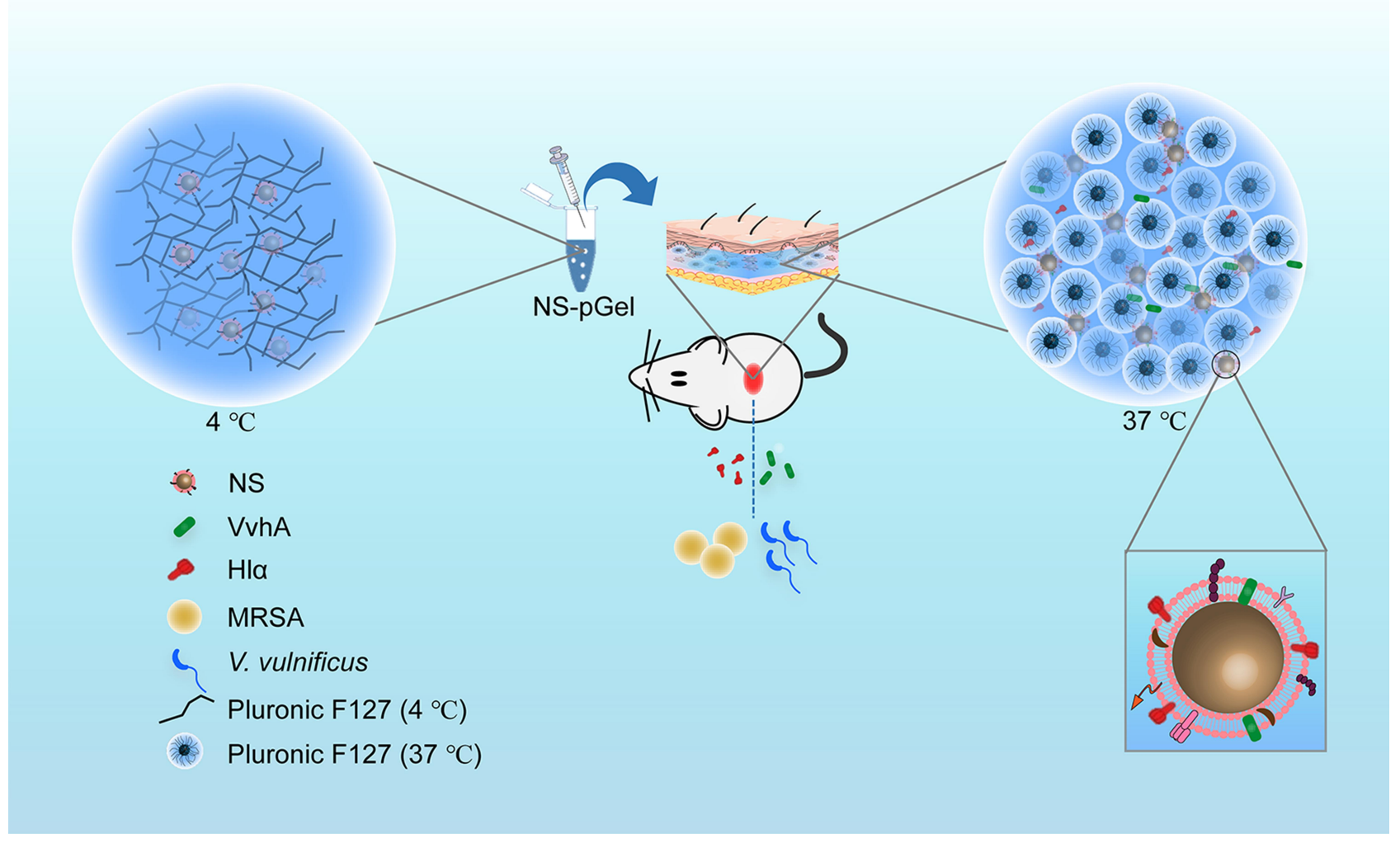

could effectively transfer pore-forming toxins (PFTs) away from their natural cellular targets and save normal cells. PFTs serve as important and abundant virulence factors from both gram-positive $\left(\mathrm{G}^{+}\right)$and gram-negative $\left(\mathrm{G}^{-}\right)$ bacterial pathogens and are well known for their ability to perforate target cells such as $\mathrm{RBCs} ;{ }^{5,11}$ thus, the NS platform with broad-spectrum absorption of PFTs is logically an effective detoxification therapy. Currently, the NS platform has been reported to inhibit the cytotoxicity of multiple PFTs, such as $\alpha$-hemolysin (Hl $\alpha$ ), streptolysin $\mathrm{O}$ (SLO) and listeriolysin O (LLO). ${ }^{12}$ However, certain drawbacks of NSs limit their further development. For example, for systemic detoxification, NSs may accumulate in the liver and cause secondary injury, especially for patients with liver failure. ${ }^{13,14}$ Further, for local application, NSs show an apparent diffusivity, thus leading to a reduction in drug levels and detoxification efficiency. ${ }^{15}$

To improve the stability and therapeutic index of NSs, incorporating NSs into hydrogels has recently gained much attention. ${ }^{16}$ Hydrogels are hydrophilic 3D crosslinked networks that can preserve the structural integrity and functions of incorporated materials. ${ }^{17}$ As advanced drug delivery reservoirs, they also have the characteristics of reduced irritation, decreased adverse effects and sustained release. ${ }^{18-22}$ Wang et $\mathrm{al}^{15}$ demonstrated the potential of nanosponge-loaded hydrogel (NS-gel) as a novel and effective detoxification strategy to treat localized methicillin-resistant Staphylococcus aureus (MRSA) infections. However, the preparation of this acrylamidebased, chemical cross-linking hydrogel requires toxic reagents, such as $\mathrm{N}, \mathrm{N}, \mathrm{N}^{\prime}, \mathrm{N}^{\prime}-$ Tetramethylethylenediamine (TEMED) and ammonium persulfate (APS), which compromise biocompatibility and limit its translational applications. In addition, the gel is not suitable for preservation because its gelation time is short, and it can only be injected when freshly prepared.

"Smart" thermosensitive hydrogels are materials displaying a sol-gel phase transition in response to 
temperature, which are superior for NS loading. ${ }^{23,24}$ Thermosensitive hydrogels maintain good liquidity and low viscosity at lower temperatures and transform into an immobile gel state immediately at body temperature. The easy injection and sustained drug release make thermosensitive hydrogels potentially useful for local drug loading and delivery. ${ }^{25-27}$ Recently, a variety of thermosensitive hydrogels have been developed, such as Pluronic F127, poly(N-isopropylacrylamide) and methylcellulose. ${ }^{28,29}$ Pluronic F127, also named Poloxamer 407, is an FDAapproved novel temperature-sensitive hydrogel material that has been used for drug delivery and wound healing. ${ }^{30-32}$ Compared to chemical cross-linking hydrogels, the pharmaceutical formulation of the Pluronic F127 hydrogel is simple and completely relies on physical methods without requiring chemical cross-linking agents, thus improving safety and reducing production costs. ${ }^{33}$ In addition, the premixed solution can sustain an aqueous state upon injection at a lower temperature with no need for fresh preparation, so it is easier to preserve. Once stimulated by near-physiological temperatures, the amphiphilic triblock copolymers in water can spontaneously form a network by physical cross-linking. These multiple advantages make the Pluronic F127 hydrogel suitable for NS incorporation.

In this study, we first incorporated NSs into FDAapproved biocompatible and thermally responsive Pluronic F127 hydrogels (denoted as NS-pGel). We then selected two representative PFTs, $V$. vulnificus hemolysin (VvhA) from $\mathrm{G}^{-} \quad V$. vulnificus and $\mathrm{Hl \alpha}$ from $\mathrm{G}^{+}$ Staphylococcus aureus for an examination of the ability for NS-pGel to absorb and neutralize these toxins. We quantified the toxin binding capacity of NS-pGel by an in vitro hemolytic assay and further confirmed the retention of NSs and the local detoxification efficiency of the NS-pGel in vivo. Overall, this study provides an injectable and novel NS-pGel as a broad-spectrum detoxification platform against different types of PFTs.

\section{Materials and Methods}

\section{Cells, Animals and Reagents}

Male ICR mice (18-20 g) were purchased from Shanghai Lab Animal Research Center. All animal experiments were performed according to the Guide for the Care and Use of Laboratory Animals and approved by Committee on Ethics of Medicine, Navy Medical University, PLA (81974496).
Carboxyl-terminated 50:50 poly (DL-lactide-coglycolide) (PLGA, Mw 48,000, $0.67 \mathrm{dL} / \mathrm{g}$ ) was purchased from Lactel Co (USA). 1,1'-Dioctadecyl-3,3,3',3'tetramethylindodicarbocyanine, 4-chlorobenzenesulfonate salt (DiD) dye was purchased from Invitrogen (USA). Pluronic F127 was obtained from Beyotime Co (Shanghai, China). Hla from Staphylococcus aureus was purchased from Sigma-Aldrich (USA). Recombinant VvhA (rVvhA) was expressed in our lab. Cell Counting Kit-8 (CCK-8) was purchased from Dojindo Molecular Technologies, Inc (Japan). All other reagents were of analytical grade, commercially available and used as received.

\section{Preparation and Characterization of NSs}

NSs were prepared by coating RBC vesicles onto PLGA nanoparticles (NPs) with reference to previous studies. ${ }^{7,34}$ Briefly, there were three steps involved in NS construction: (i) Whole blood withdrawn from male ICR mice (20$25 \mathrm{~g}$ ) was centrifuged at $1000 \mathrm{rpm}$ for $15 \mathrm{~min}$ at $4{ }^{\circ} \mathrm{C}$ and then washed 3 times until the plasma and buffy coat were completely removed. Next, the resulting RBCs were lysed by a hemolytic protocol followed by high-speed centrifugation at $12,000 \mathrm{rpm}$ for $20 \mathrm{~min}$. The sediment was washed repeatedly to harvest a white pellet. The resulting RBC ghosts were then serially extruded through 400 and $200 \mathrm{~nm}$ polycarbonate porous membranes to obtain $\mathrm{RBC}$ vesicles. (ii) PLGA NPs were prepared by the nanoprecipitation method. ${ }^{7}$ PLGA polymers $(5 \mathrm{mg} / \mathrm{mL})$ dissolved in acetone $(1 \mathrm{~mL})$ were poured into distilled water $(3 \mathrm{~mL})$ and stirred until the acetone was completely evaporated. A similar method was used to prepare DiD-labelled PLGA NPs, except that $0.1 \%(\mathrm{w} / \mathrm{w})$ DiD fluorescence dye was added to the PLGA acetone solution. (iii) The RBC vesicles were mixed with PLGA NPs at a protein-to-polymer weight ratio of $1: 1$, and then NSs were constructed by the coextrusion method. ${ }^{7}$

The hydrodynamic size and zeta potential of prepared RBC vesicles, PLGA NPs and NSs were measured by dynamic light scattering (DLS) at room temperature. The structure of the NSs was examined using transmission electron microscopy (TEM) (JEOL JEM-2010, Japan) by negative staining with $1 \%$ uranyl acetate. The cell membrane proteins retained on the NSs were characterized by sodium dodecyl sulfate-polyacrylamide gel electrophoresis (SDS-PAGE), and RBC ghosts and vesicles were used as controls. First, the protein concentration of all samples was determined by the bicinchoninic acid (BCA) protein assay 
kit (Thermo Scientific, Pierce, Shanghai). Then, all the samples were heated to $100{ }^{\circ} \mathrm{C}$ for $15 \mathrm{~min}$, and $20 \mu \mathrm{L}$ of samples with equal protein amounts were added and run on a $6 \%$ stacking gel at $80 \mathrm{~V}$ for $25 \mathrm{~min}$ and separation gel at $120 \mathrm{~V}$ for another $1 \mathrm{~h}$ using a BIO-RAD ${ }^{\circledR}$ Electrophoresis System. Finally, the resulting gel was stained with Coomassie Blue (Sangon, China) for imaging.

\section{Preparation of Pluronic FI27 Hydrogel, NS-pGel and the Sol-Gel Transition}

For bare hydrogel preparation, Pluronic F127 solutions of different concentrations $(10 \%, 15 \%, 20 \%, 25 \%, 30 \%)$ were prepared by dissolving Pluronic F127 powder into cold distilled water. The mixture was then magnetically stirred at 4 ${ }^{\circ} \mathrm{C}$ for complete dissolution, and the bubbles were removed overnight. For NS-pGel preparation, NSs at a final concentration of $1 \mathrm{mg} / \mathrm{mL}$ were added to $30 \%$ Pluronic F127 precursor solution at $4{ }^{\circ} \mathrm{C}$ followed by moderate stirring and bubble removal. At $37{ }^{\circ} \mathrm{C}$, the NS-pGel composite was formed in situ due to the temperature-induced sol-gel transition. Samples of Pluronic F127 hydrogel and NS-pGel were examined and photographed after gel formation.

\section{Characterization of the Pluronic FI27 Hydrogel and NS-pGel}

The NS-pGel and Pluronic F127 hydrogel were characterized with dynamic rheological measurements on a rheometer (Anton Paar, Germany). The storage modulus $\left(\mathrm{G}^{\prime}\right)$ and loss modulus $\left(\mathrm{G}^{\prime \prime}\right)$ were measured as a function of temperature sweeping from $10{ }^{\circ} \mathrm{C}$ to $65^{\circ} \mathrm{C}$ at an angular frequency of $1 \mathrm{rad} / \mathrm{s}$ to determine the phase transition temperature of the NS-pGel. Then, $\mathrm{G}^{\prime}$ and $\mathrm{G}^{\prime \prime}$ were measured as a function of frequency ranging from $0.1 \mathrm{~Hz}$ to 10 $\mathrm{Hz}$ at $25{ }^{\circ} \mathrm{C}$ to examine the gel formation. In addition, the viscosity of the NS-pGel was tested to determine the injectability at lower temperatures.

To test the release rate of NSs in the hydrogel, $0.5 \mathrm{~mL}$ of DiD-labelled NS-pGel was immersed in $5 \mathrm{~mL}$ of PBS buffer and incubated at $37^{\circ} \mathrm{C}$. At a predetermined time $(0,1,2,4,8$, $12,24,48 \mathrm{~h}$ ), all the buffer was withdrawn and replaced with $5 \mathrm{~mL}$ of fresh buffer solution. Then, the fluorescence intensity of DiD was measured by a Varioskan Flash Multimode Reader (Thermo Scientific Company, USA), and the ratio of released NSs was calculated.

To examine the morphology, NS-pGel was lyophilized and coated with a thin layer of gold before observation under a scanning electron microscope (SEM).
For biocompatibility examination, L929 cells grown in 96-well plates were treated with leaching liquor $(25 \%$, $50 \%$ and $100 \%$ ) from NS-pGel for 24 and $48 \mathrm{~h}$, and cell viability was determined by a CCK- 8 kit.

\section{PFT Neutralization Study of NS-pGel in vitro}

Initially, the activities of $\mathrm{rVvhA}$ and $\mathrm{Hl} \alpha$ were determined by a hemolysis protocol in a $2 \%$ murine RBC suspension. Then, the in vitro toxin neutralization ability of NSs was examined by mixing NSs of various concentrations $(0.016 \sim 2 \mathrm{mg} / \mathrm{mL}$, a final volume of $0.5 \mathrm{~mL})$ with $\mathrm{rVvhA}$ $(15 \mu \mathrm{g} / \mathrm{mL})$ or Hla $(1 \mu \mathrm{g} / \mathrm{mL})$. Next, an equal volume of the $2 \% \mathrm{RBC}$ suspension was added and incubated at $37^{\circ} \mathrm{C}$ for $30 \mathrm{~min}$. Subsequently, the samples were centrifuged at $1000 \mathrm{rpm}$ for $5 \mathrm{~min}$, and the released hemoglobin was quantified at $540 \mathrm{~nm}$ to determine the degree of hemolysis using a Varioskan Flash Multimode Reader. Furthermore, a similar hemolysis protocol was used to evaluate the toxin neutralization ability of NS-pGel (containing $1 \mathrm{mg} / \mathrm{mL}$ of NSs). Briefly, rVvhA $(15 \mu \mathrm{g} / \mathrm{mL})$ or Hla $(1 \mu \mathrm{g} / \mathrm{mL})$ was added to NS-pGel, gel, NS and PBS, respectively (a final volume of $0.5 \mathrm{~mL}$ ). After incubation for gelation at $37{ }^{\circ} \mathrm{C}$, an equal volume of the $2 \% \mathrm{RBC}$ suspension was added and incubated at $37{ }^{\circ} \mathrm{C}$ for $30 \mathrm{~min}$. Each sample was then carefully centrifuged for hemolysis examination.

\section{Live Imaging for NS Retention in vivo}

Prior to the study, the back of the male ICR mice (18-20 g) was carefully shaved. Then, $50 \mu \mathrm{L}$ of DiD-labelled NSpGel was injected subcutaneously into the left flank of the mice. As a control, $50 \mu \mathrm{L}$ of DiD-labelled NSs was injected subcutaneously into the right flank of the same mice. At the designated time points $(0,12,24,48 \mathrm{~h})$, the mice were anesthetized with sevoflurane and imaged with a QuickView3000 (Bio-Real) system. Fluorescence intensities were then quantified.

\section{PFT Neutralization Study of NS-pGel in vivo}

The experiment was performed as previously described. ${ }^{15}$ First, $30 \mu \mathrm{g}$ of $\mathrm{rVvhA}$ or $2 \mu \mathrm{g}$ of Hl $\alpha$ solution $(50 \mu \mathrm{L})$ was injected into the flanks of male ICR mice (18-20 g). Then, $100 \mu \mathrm{L}$ samples of NS, NS-pGel or saline were injected into the same wound site. After treatment for $72 \mathrm{~h}$, the lesions were imaged and measured, and then the mice were anesthetized for harvesting skin and muscle samples. 
The samples were immobilized and stained with hematoxylin and eosin (H\&E staining) for pathological examination under a microscope.

We further explored the prophylactic detoxification efficiency of NS-pGel in vivo. NS or NS-pGel samples were injected into the flank subcutaneously $24 \mathrm{~h}$ prior to $30 \mu \mathrm{g}$ of $\mathrm{rVvhA}$ or $2 \mu \mathrm{g}$ of Hla exposure. The remaining steps were repeated as described above.

\section{Statistical Analysis}

All the measurements were performed in triplicate. The results were expressed as the mean \pm standard error (SE). Data were analyzed by one-way ANOVA. Differences were considered statistically significant at $P<0.05(*)$.

\section{Results}

\section{Characterization of NS}

To characterize the hydrodynamic size and zeta potential, the PLGA NPs and RBC-derived NSs were measured by DLS. The results revealed an increase $(\sim 15 \mathrm{~nm})$ in the average hydrodynamic diameters of NPs, which corresponded to RBC membrane wrapping on NPs (Figure 1A). Meanwhile, the zeta potential of the PLGA NPs increased from $-37.5 \pm 2.3$ to $-24.1 \pm 1.9 \mathrm{mV}$, which was close to the surface potential of the RBC membrane (Figure 1B). Together, these results demonstrated fusion between NP cores and RBC membranes.

To characterize the nanostructures, the resulting NS sample was dried and examined by TEM. The ultrastructure exhibited a typical core-shell structure with a polymer core coated by a membrane shell, revealing the formation of NSs (Figure 1C).

To further characterize the protein content retained on the NSs, the resulting NS sample was analyzed by SDSPAGE. The gel image indicated that most of the membrane proteins were retained on the NSs after preparation, which was consistent with those of RBC ghosts and vesicles (Figure 1D).

Overall, the results of physiological characterization and protein retention examination collectively suggested the successful construction of NSs.

\section{Optimization of Hydrogel and NS-pGel Preparation}

To achieve optimal gel formation, Pluronic F127 solutions of various concentrations were screened. As shown in Figure 2A, each group of Pluronic F127 solutions maintained good fluidity at $4{ }^{\circ} \mathrm{C}$, and as the concentration of Pluronic F127 solution increased, the gelation capacity was enhanced at $37{ }^{\circ} \mathrm{C}$. The Pluronic F127 solution with a concentration below $25 \%$ was not capable of forming hydrogels at body temperature, while the $30 \%$ gel solution showed an optimal gel state. Thus, we chose $30 \%$ gel solution to load NS (1 mg/mL). Notably, NS-pGel showed a muddy appearance because of the addition of NS suspension, but the fluidity at $4{ }^{\circ} \mathrm{C}$ and gelation at $37^{\circ} \mathrm{C}$ were not influenced after mixing (Figure 2B). The above results indicate that the NS-pGel hybrid detoxification system is convenient for storage thanks to everlasting fluidity at lower temperatures, and it permits quick gelation at body temperature.

\section{Characterization of the Pluronic FI27 Hydrogel and NS-pGel}

The NS-pGel was characterized with dynamic rheological measurements of the storage modulus $\left(\mathrm{G}^{\prime}\right)$ and the loss modulus $\left(\mathrm{G}^{\prime \prime}\right)$ as a function of temperature. As shown in Figure $3 \mathrm{~A}, \mathrm{G}^{\prime}$ and $\mathrm{G}^{\prime \prime}$ dramatically increased at approximately $25{ }^{\circ} \mathrm{C}$, ie, gelation could quickly occur at this temperature. In addition, $\mathrm{G}^{\prime}$ exceeded $\mathrm{G}^{\prime \prime}$ over the entire frequency range at $37{ }^{\circ} \mathrm{C}$, indicating typical viscoelastic behavior and the stability of the formed hydrogel system (Figure 3B). Viscosity examination results revealed that the viscosity of NS-pGel fluctuated approximately $0 \mathrm{~Pa} \cdot \mathrm{s}$ below $15{ }^{\circ} \mathrm{C}$ but increased with heating and reached a plateau at approximately $30^{\circ} \mathrm{C}$, indicating that the NSpGel is easily injected by syringe at lower temperatures and can sustain a high-viscosity gel state for NS retention at body temperature (Figure 3C).

Next, we used fluorescence detection to verify the NS release properties of NS-pGel. As shown in Figure 3D, the release of NSs and the degradation of the hydrogel were time-dependent. Specifically, the release rate of NSs at 1 h was approximately $10 \%$, which gradually increased to $90 \%$ at $48 \mathrm{~h}$. Similarly, gel degradation was lower than 5\% at $1 \mathrm{~h}$ and gradually increased to $70 \%$ at $48 \mathrm{~h}$. These results revealed that the NS-pGel hybrid system showed a sustained release behavior accompanied by slow degradation of the Pluronic F127 hydrogel.

To further characterize the nanostructure, freeze-dried NS-pGel and blank hydrogel samples were observed by SEM. The images showed typical porous sponge-like structures of gel materials, further revealing the formation of a hybrid hydrogel system. Notably, at a higher 
A

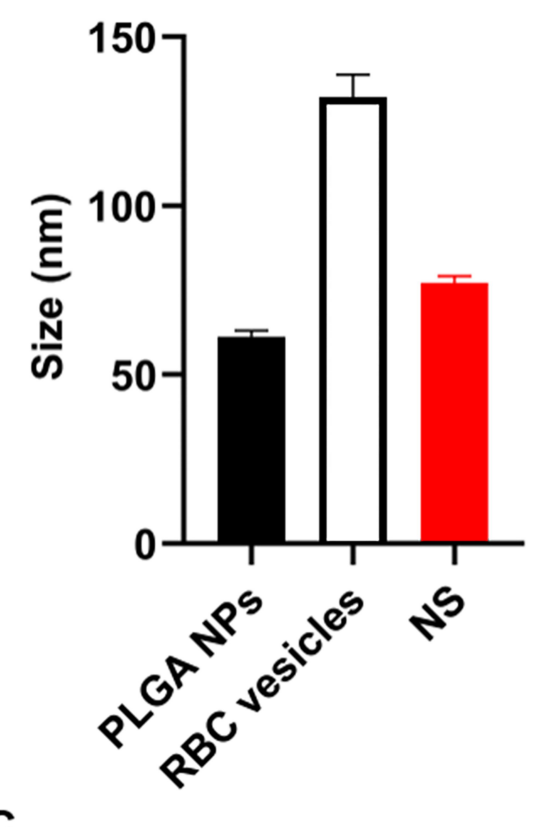

C

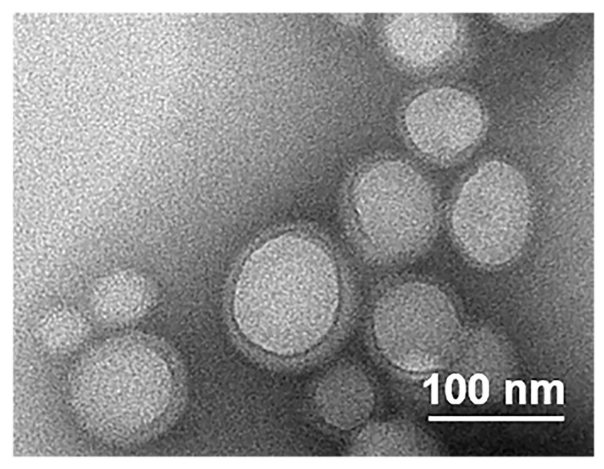

$\mathbf{B}$

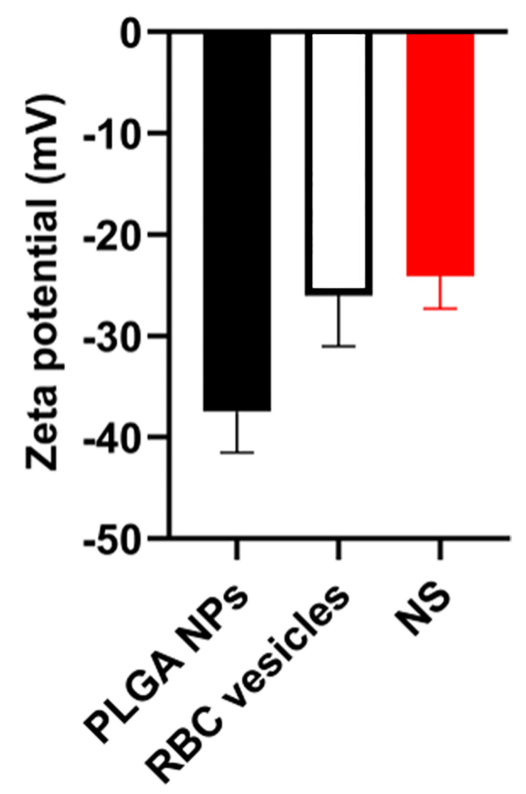

D

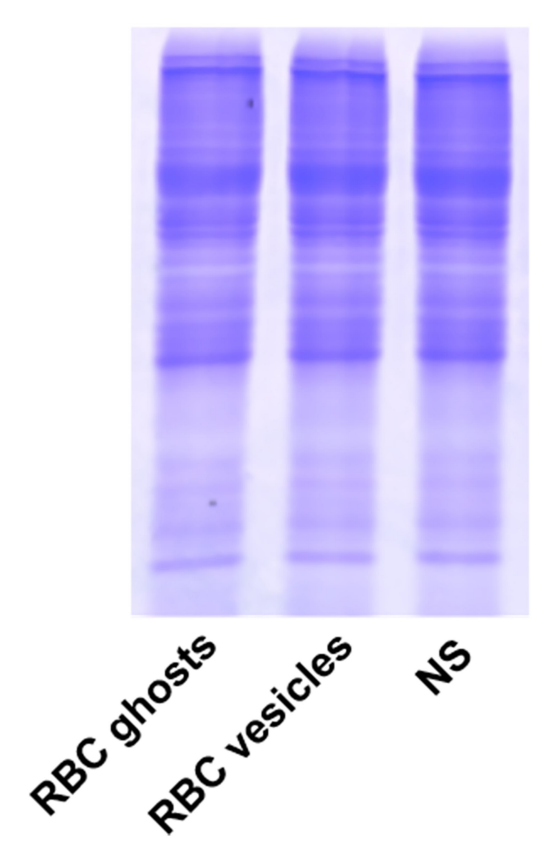

Figure I Characterization of NSs. (A-B) Hydrodynamic size and zeta potential of PLGA NPs, RBC vesicles and NSs detected by DLS. (C) A representative TEM image demonstrates the core-shell structure of NSs with RBC membrane shells wrapped onto core NPs. (D) Proteins on RBC ghosts, RBC vesicles, and NSs resolved on a polyacrylamide gel were examined by SDS-PAGE.

magnification, nanosponges loaded in hydrogel were also observed (Figure 3E).

To test the safety of NS-pGel, L929 cells were treated with various doses of NS-pGel for 24 and $48 \mathrm{~h}$. As shown in Figure 3F, the cell survival rates of all the samples were over $80 \%$, revealing the nonpoisonous and compatible characteristics of NS-pGel.

\section{PFT Neutralization of NSs and NS-pGel in vitro}

Hemolysis experiments were performed to determine the optimal dose of PFTs for subsequent study. As shown in Figure 4A and $\mathrm{D}$, the ratio of hemolysis enhanced in an "S" shape with increasing concentrations of PFT toxins ( $\mathrm{rVvhA}$ and $\mathrm{Hl} \alpha$ ), which reached peaks at doses of $15 \mu \mathrm{g} / \mathrm{mL}(\mathrm{rVvhA})$ and 1 
A
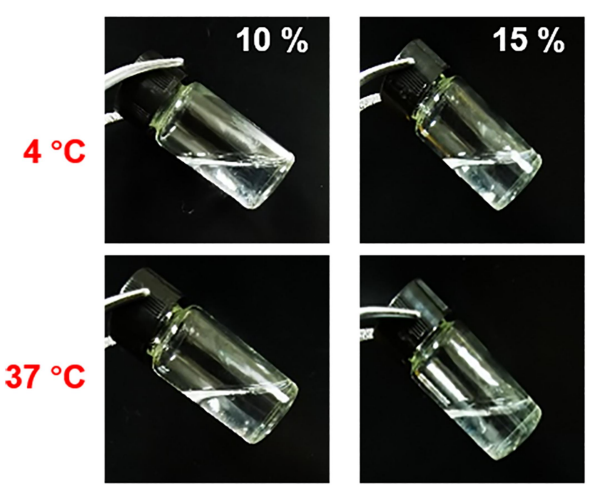
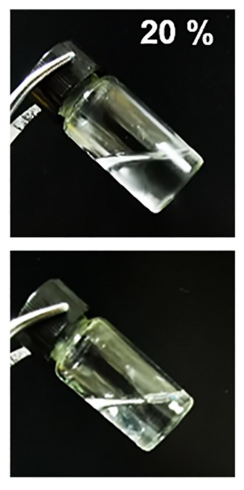

Pluronic F127 hydrogel
B
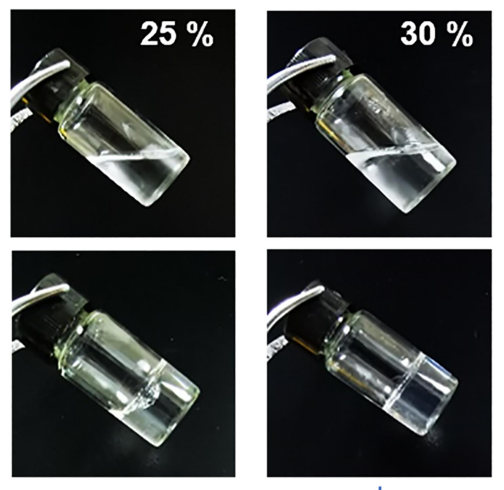

J

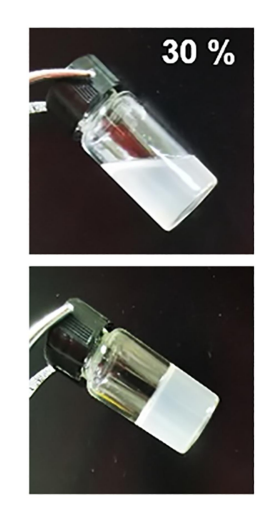

NS-pGel

Figure 2 Optimization of Pluronic FI 27 hydrogel and NS-pGel. (A) Gel resolution of different concentrations (10\%, $15 \%, 20 \%, 25 \%$ and $30 \%$ ) at $4{ }^{\circ} \mathrm{C}$ (upper image) and gel formation of Pluronic FI27 at $37{ }^{\circ} \mathrm{C}$ (lower image). (B) NS-pGel resolution at $4{ }^{\circ} \mathrm{C}$ (upper image) and gel formation at $37{ }^{\circ} \mathrm{C}$ (lower image).

A

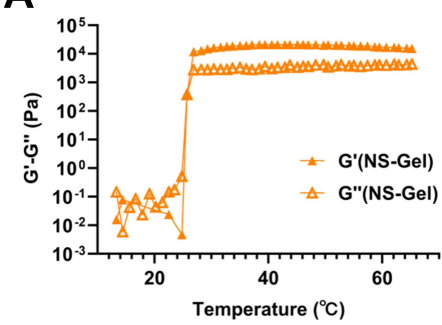

D

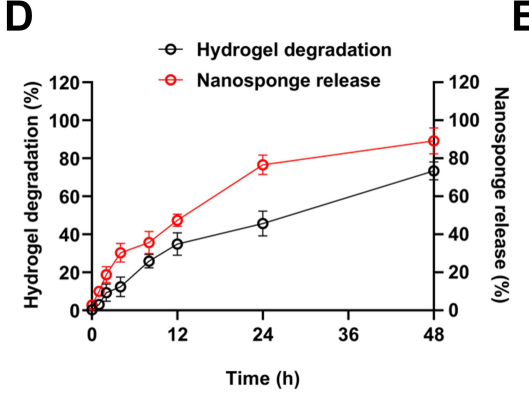

B

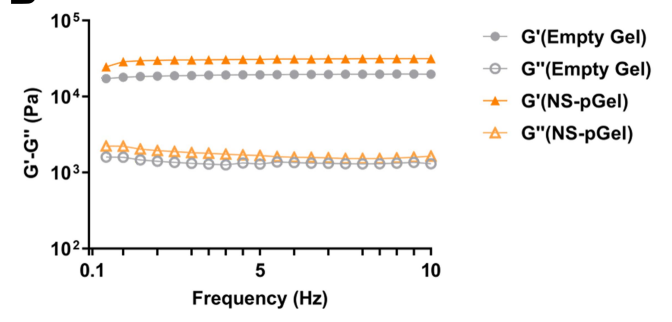

E

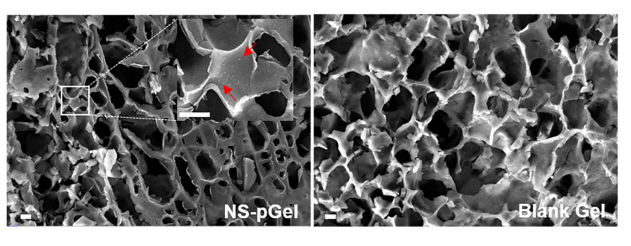

C

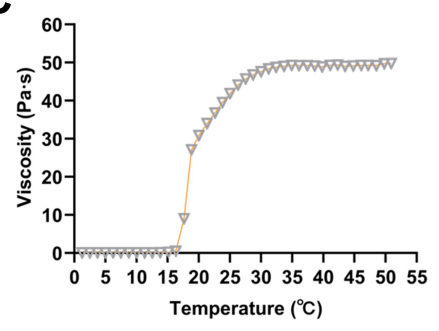

F

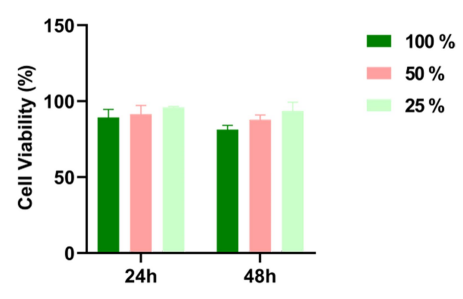

Figure 3 Characterization of the Pluronic FI 27 hydrogel and NS-pGel. (A) Rheological characterization of the Pluronic FI27 hydrogel (30\%) loaded with I mg/mL NS. The storage modulus $\mathrm{G}^{\prime}$ and loss modulus $\mathrm{G}^{\prime \prime}$ were plotted logarithmically against temperature $\left(10 \sim 70^{\circ} \mathrm{C}\right)$. (B) Rheological characterization of $\mathrm{NS}$-pGel as a function of frequency $(0.1 \sim 10 \mathrm{~Hz}$ at $\left.37^{\circ} \mathrm{C}\right)$. (C) Viscosity of NS-pGel determined against temperatures between 0 and $55^{\circ} \mathrm{C}$. (D) Degradation and NS release kinetics of NS-pGel detected over a period of I 20 h. (E) Representative SEM images of NS-pGel (left image, red arrows refer to NS) and blank hydrogel (right image) (Scale bar: $2 \mu \mathrm{m})$. (F) Cytotoxic effect of NS-pGel on L929 cells. Cells were pretreated with leaching liquor (100\%, 50\% and $25 \%$ ) for $24 \mathrm{~h}$ or $48 \mathrm{~h}$. Cell viability was determined by a CCK-8 kit. ( $\mathrm{n}=3$ ).

$\mu \mathrm{g} / \mathrm{mL}(\mathrm{Hl} \alpha)$, respectively. When treated with NSs, the detoxification capacity was enhanced with the increase in these antidotes. The $0.5 \mathrm{mg} / \mathrm{mL}$ concentration of NSs almost completely inhibited hemolysis induced by $15 \mu \mathrm{g} / \mathrm{mL}$ of $\mathrm{rVvhA}$ (Figure 4B). Similarly, the hemolysis induced by $1 \mu \mathrm{g} / \mathrm{mL}$ of Hl $\alpha$ was almost completely reversed by the $1 \mathrm{mg} / \mathrm{mL}$ of NS sample (Figure 4E). Next, the hemolysis-inhibition effect of NS-pGel (loaded with $1 \mathrm{mg} / \mathrm{mL}$ of NS) was examined. Our results showed that when compared to NSs, NS-pGel inhibited hemolysis caused by PFTs (rVvhA, Hla) to a similar extent, but the hydrogel failed to show a protective effect alone (Figure $4 \mathrm{C}$ and $\mathrm{F}$ ). These results indicate that gel formulation preserves the critical biological functionality of NSs.

\section{The Pluronic FI27 Hydrogel Retained NSs Locally in vivo}

We then evaluated the retention of NSs by the Pluronic F127 hydrogel in vivo. Within $48 \mathrm{~h}$, the fluorescence 

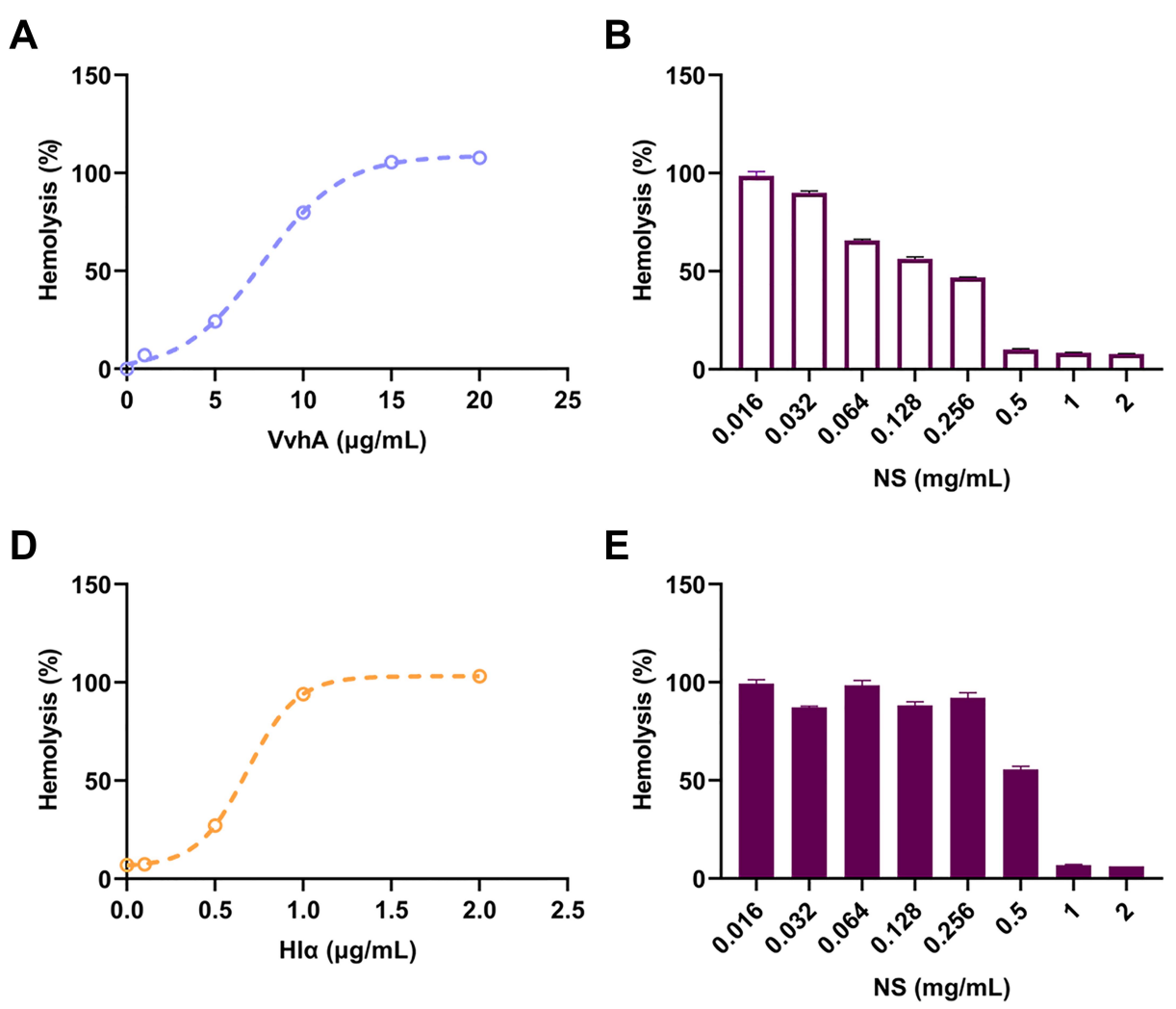
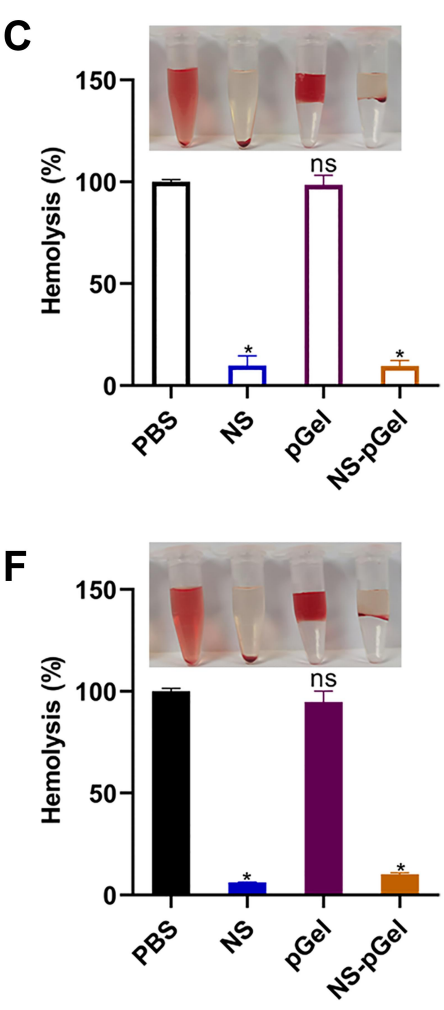

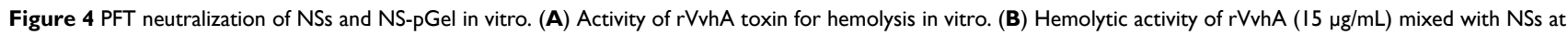
different concentrations. (C) Centrifuged RBCs and hemolysis quantification after incubation with rVvhA (I5 $\mu \mathrm{g} / \mathrm{mL}) \mathrm{mixed}$ with PBS, NSs (I mg/mL), empty gel and NS-pGel (containing I mg/mL NS). (D) Hemolytic activity of Hl $\alpha$ toxin in vitro. (E) Hemolysis caused by Hla $(\mathrm{I} \mu \mathrm{g} / \mathrm{mL}$ ) mixed with NSs at different concentrations. (F) Centrifuged RBCs and hemolysis quantification after incubation with Hla mixed with PBS, NSs, empty gel and NS-pGel. (Data represent the mean \pm SE, $n=3$, $* P<0.05$, ns: no significance).

intensity of the NS group showed a more rapid decay than that of the NS-pGel group (Figure 5A). Quantification analysis revealed that over $90 \%$ of the NSs diffused over a period of $48 \mathrm{~h}$, while only approximately $40 \%$ of the NSs were lost in the NS-pGel group (Figure 5B), indicating good NS retention in the hydrogel.

\section{Inhibition Effects of NS-pGel on PFTs in vivo}

The efficiency of NS-pGel in neutralizing PFTs in vivo was further examined by subcutaneous injection of various PFTs (rVvhA, Hla) in mice. After $72 \mathrm{~h}$, mice exposed to rVvhA $(30 \mu \mathrm{g})$ or Hl $\alpha(2 \mu \mathrm{g})$ and treated with saline showed obvious
A

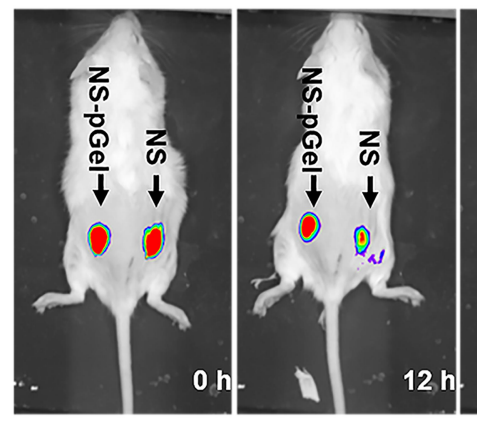

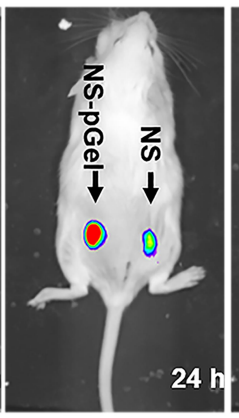

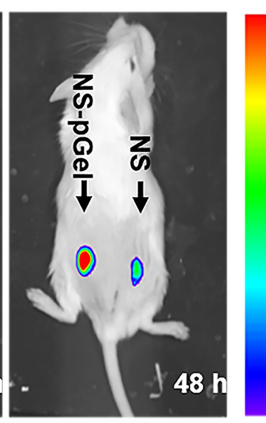

B

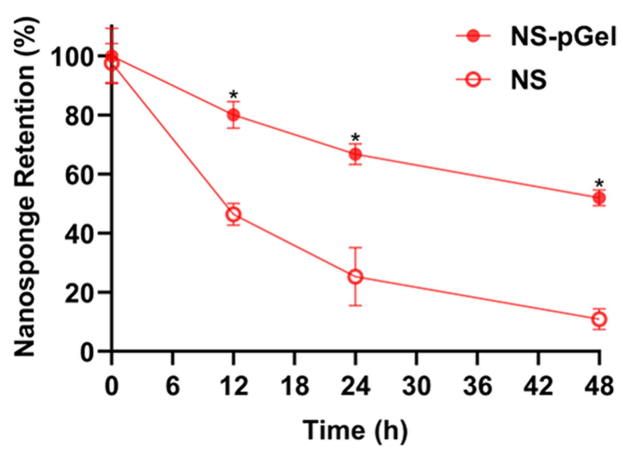

Figure 5 Local NS retention by Pluronic FI 27 hydrogel. DiD-labelled NSs were injected subcutaneously over the right flanks of mice. DiD-labelled NSs retained by hydrogel (NS-pGel) were injected into the left flanks of the same mice. (A) Fluorescence images were taken at certain time points to determine the NS retention in vivo. (B) Relevant quantification of the fluorescence intensity. (Data represent the mean $\pm \mathrm{SE}, \mathrm{n}=3, * \mathrm{P}<0.05$ ). 

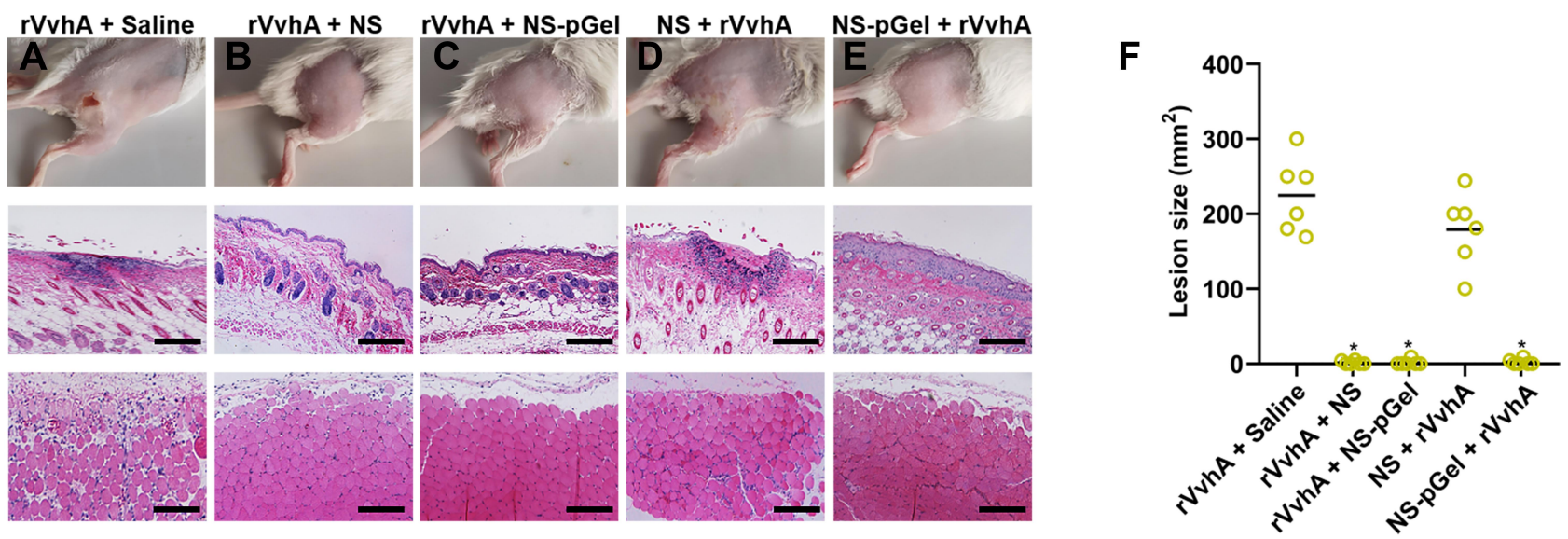

Figure 6 rVvhA neutralization efficiency of NS-pGel in vivo. (A-C) Mice were injected with rVvhA into the flank region immediately followed by saline, NSs or NS-pGel. The local lesions were photographed after $72 \mathrm{~h}$, and then the skin and muscle were harvested for H\&E staining and histological examination. (D-E) Mice were injected with saline, NSs or NS-pGel subcutaneously into the flanks $24 \mathrm{~h}$ before $\mathrm{rVvhA}$ exposure. Images of lesions and H\&E-stained tissues were obtained after $72 \mathrm{~h}$. (scale bar: 50 um). (F) Skin lesions of each group were measured after treatment. (Data represent the mean $\pm S E, n=6, * P<0.05$ versus group treated with saline).

skin lesions with reddish ecchymosis and inflammation. Histological changes also appeared in the skins and muscles, including apparent oedema, cell death, as well as neutrophil accumulation (Figures 6A, F and 7A, F). However, both the NS ( $2 \mathrm{mg}$ ) and NS-pGel (equal to $2 \mathrm{mg}$ of NS) markedly diminished PFT-induced tissue damage and inflammatory reactions (Figures 6B, C, F and 7B, C, F). Furthermore, the mice pretreated with NS-pGel suffered milder local lesions than those pretreated with NS alone (Figures 6D-F and 7DF). Therefore, it can be concluded that NS-pGel possesses both therapeutic and preventive effects.

\section{Discussion}

Recently, therapeutic biomimetic nanosponges have been increasingly combined with other biomaterials to form stable structures to improve the therapeutic effects against infectious diseases. In this study, we successfully used RBC-derived NSs and Pluronic F127 hydrogel to construct a novel biocompatible and biodegradable detoxification system denoted "NS-pGel", which is formed entirely based on physical cross-linking instead of chemical crosslinking. NS-pGel not only preserves the toxin neutralization capability of the incorporated NSs but also achieves a significantly prolonged retention of NSs in both biological buffers and mouse subcutaneous tissues. More importantly, this hybrid hydrogel system has typical temperature-sensitive characteristics with injectable ability at lower temperatures and quick gelation near body temperature and is thus suitable for local wounds with various shapes. Together, these multiple advantages make this
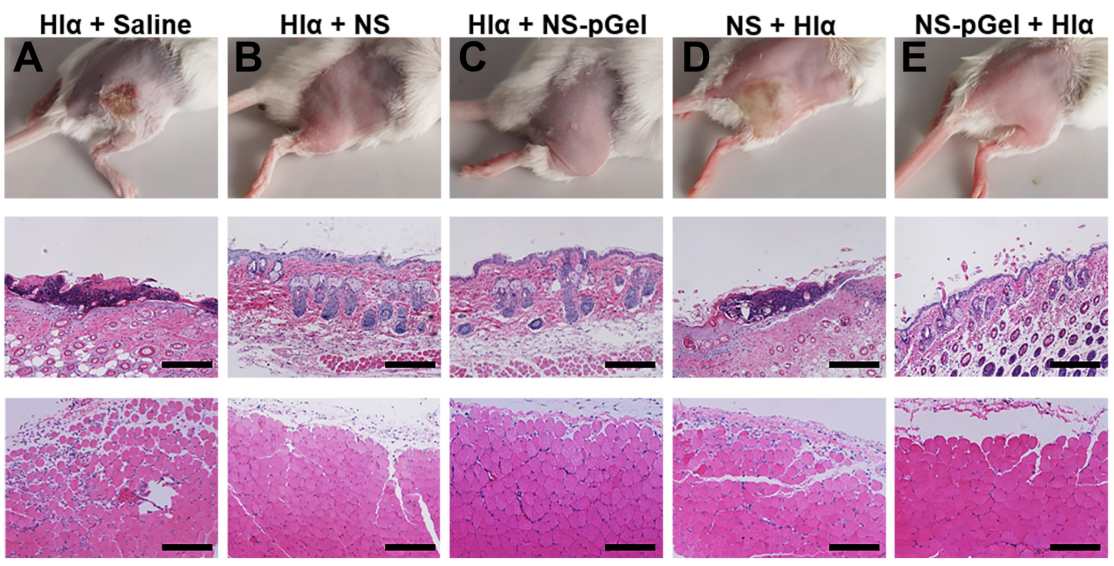

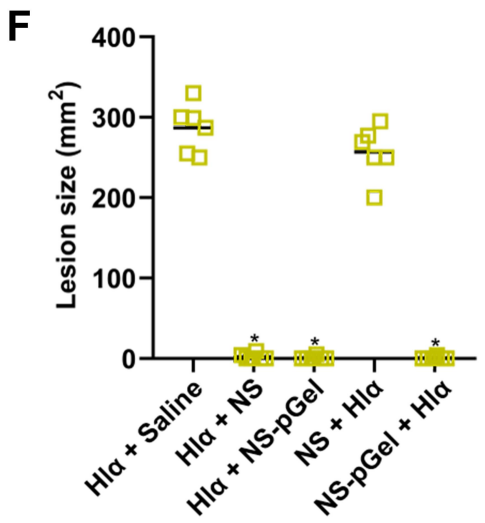

Figure $7 \mathrm{Hl} \alpha$ neutralization efficiency of NS-pGel in vivo. (A-C) Mice were injected with Hla into the flank region immediately followed by saline, NSs or NS-pGel. The local lesions were photographed after $72 \mathrm{~h}$, and then the skin and muscle were harvested for H\&E staining and histological examination. (D-E) Mice were injected with saline, NSs or NS-pGel subcutaneously into the flanks $24 \mathrm{~h}$ before Hl $\alpha$ exposure. Images of lesions and H\&E-stained tissues were obtained after $72 \mathrm{~h}$. (scale bar: $50 \mu \mathrm{m}$ ). (F) Skin lesions of each group were measured after treatment. (Data represent the mean $\pm S E, n=6, * P<0.05$ versus group treated with saline). 
"NS-pGel" a promising injectable drug reservoir for medical applications, including anti-virulence therapy against localized bacterial infection.

In this study, we employed a Pluronic F127 hydrogel to incorporate NSs for the construction of a nanospongehydrogel hybrid system for the first time. As previously mentioned, Pluronic F127 is an FDA-approved thermosensitive hydrogel that has been adopted for drug delivery and wound healing. Based on physical cross-linking, the preparation of Pluronic F127 hydrogel is apparently simple and occurs by resolving and stirring, thus providing the possibility for large-scale production and clinical transformation. In the present study, we chose a $30 \%$ Pluronic F127 hydrogel solution to construct NS-pGel because the resulting detoxification system had a good fluidity at $4{ }^{\circ} \mathrm{C}$ and a stable gel state at body temperature (Figure 2). In addition, viscosity examination also revealed that the viscosity of NS-pGel was significantly low at temperatures below $15{ }^{\circ} \mathrm{C}$ but dramatically increased near body temperature. Together, the temperature-sensitive properties of NS-pGel make it easily injectable by syringe, and longterm storage is possible instead of spontaneous gelation at lower temperatures. More importantly, compared to chemical cross-linking, the preparation of NS-pGel is physical, simple, and chemical-free, allowing NS-pGel to retain the functionalities of NSs without affecting the original functions. ${ }^{35}$

For local administration and treatment, the prolonged retention of NSs by using a hydrogel is desirable. In this study, NS-pGel was formulated with fluorescence-labelled NSs, and the release of NSs was monitored by measuring the fluorescence intensity in vitro. NS-pGel released $10 \%$ of the total NS amount in $1 \mathrm{~h}$, with the release lasting for nearly 48 $\mathrm{h}$, indicating that the NS-pGel system can effectively immobilize and retain NSs within its network (Figure 3D). We then investigated the retention of NSs within the pGel in vivo. Whole-body imaging of the mice and quantification of the fluorescence intensity revealed the confinement and a negligible loss of NSs at sites injected with NS-pGel compared to those injected with free NS (Figure 5), demonstrating that the prolonged retention of NSs was achieved with the NS-pGel formulation. Simultaneously, we also found that the prepared NS-pGel possessed good biocompatibility and safety (Figure 3F), further demonstrating its significant potentials as a local treatment agent to be used on human bodies in the future.

Finally, the NS-pGel system showed a significant detoxification efficiency both in vitro and in vivo. Since antibiotic-resistant $\mathrm{G}^{+} / \mathrm{G}^{-}$bacterial infections pose a threat to humans, we chose two well-characterized PFTs, Hla from $\mathrm{G}^{+}$Staphylococcus aureus and VvhA from $\mathrm{G}^{-} V$. vulnificus, to examine the detoxification ability of the NS-pGel system compared to free NSs. In vitro hemolysis examination revealed that NS-pGel samples showed a similar inhibition efficiency compared to that of free NSs (Figure 4C and F), suggesting that the NSpGel formulation retains the full neutralization capability of its component NSs. Then, neutralization of PFTs by NS-pGel was further tested in vivo. Similar toxin neutralization effects were observed in the NS-pGel group compared to the NS group, further confirming that the neutralization function of NSs was well preserved within the NS-pGel formulation. Finally, to demonstrate the prophylactic detoxification potential of NS-pGel, we injected NS-pGel $24 \mathrm{~h}$ before toxin exposure. As a result, NS-pGel showed better preventive effects than NSs (Figures 6 and 7). We speculate that the main reason is the sustained release of NSs from the NS-pGel system, which causes retention of NSs in wounds at a high concentration. Overall, the Pluronic F127 hydrogel endows NSs with the possibility of therapeutic and prophylactic application.

\section{Conclusion}

In conclusion, the present study developed a novel detoxification system (denoted NS-pGel) with good injectability and temperature sensitivity. Biocompatible Pluronic F127 hydrogel was incorporated with NSs for the first time, and the hydrogel effectively retained NSs within its network without compromising their toxin neutralization capability. Ex vivo, NS-pGel exhibited an excellent efficacy on hemolysis inhibition caused by PFTs; in a mouse model of subcutaneous infection, NS-pGel also showed a significant alleviation of skin lesion development in both therapeutic and prophylactic settings. This work may lead to novel clinical treatments for PFT-induced local lesions and infections.

\section{Abbreviations}

NS, nanosponge; NS-pGel, nanosponge-loaded Pluronic F127 hydrogel; PFT, pore-forming toxin.

\section{Author Contributions}

All authors made substantial contributions to conception and design, acquisition of data, or analysis and interpretation of data; took part in drafting the article or revising it critically for 
important intellectual content; agreed to submit to the current journal; gave final approval of the version to be published, and agreed to be accountable for all aspects of the work.

\section{Funding}

The authors thank the National Key R\&D Program of China (2019YFC0312605) and the National Natural Science Foundation of China (81974496) for financial support.

\section{Disclosure}

The authors report no conflicts of interest in this work.

\section{References}

1. Morris S, Cerceo E. Trends, epidemiology, and management of multi-drug resistant gram-negative bacterial infections in the hospitalized setting. Antibiotics (Basel). 2020;9(4):196. doi:10.3390/ antibiotics 9040196

2. Genovese C, La Fauci V, D'Amato S, et al. Molecular epidemiology of antimicrobial resistant microorganisms in the 21th century: a review of the literature. Acta Biomed. 2020;91(2):256-273. doi:10.23750/abm.v91i2.9176

3. Christaki E, Marcou M, Tofarides A. Antimicrobial resistance in bacteria: mechanisms, evolution, and persistence. $J$ Mol Evol. 2020;88(1):26-40. doi:10.1007/s00239-019-09914-3

4. Bielecki J, Youngman P, Connelly P, Portnoy DA. Bacillus subtilis expressing a haemolysin gene from Listeria monocytogenes can grow in mammalian cells. Nature. 1990;345:175-176. doi:10.1038/ $345175 \mathrm{a} 0$

5. Los FC, Randis TM, Aroian RV, Ratner AJ. Role of pore-forming toxins in bacterial infectious diseases. Microbiol Mol Biol Rev. 2013;77(2):173-207.

6. Kho MF, Bellier A, Balasubramani V, et al. The pore-forming protein Cry5B elicits the pathogenicity of Bacillus sp. against Caenorhabditis elegans. PLoS One. 2011;6(12):e29122. doi:10.1371/journal. pone. 0029122

7. Hu CMJ, Zhang L, Aryal S, Cheung C, Fang RH, Zhang L. Erythrocyte membrane-camouflaged polymeric nanoparticles as a biomimetic delivery platform. Proc Natl Acad Sci. 2011;108 (27):10980-10985. doi:10.1073/pnas.1106634108

8. Fang RH, Luk BT, Hu CM, Zhang L. Engineered nanoparticles mimicking cell membranes for toxin neutralization. Adv Drug Deliv Rev. 2015;90:69-80. doi:10.1016/j.addr.2015.04.001

9. Chen Y, Zhang Y, Chen M, et al. Biomimetic nanosponges suppress in vivo lethality induced by the whole secreted proteins of pathogenic bacteria. Small. 2019;15(6):e1804994. doi:10.1002/smll.201804994

10. Hu CM, Fang RH, Copp J, Luk BT, Zhang L. A biomimetic nanosponge that absorbs pore-forming toxins. Nat Nanotechnol. 2013;8 (5):336-340. doi:10.1038/nnano.2013.54

11. Bischofberger M, Iacovache I, van der Goot FG. Pathogenic pore-forming proteins: function and host response. Cell Host Microbe. 2012;12(3):266-275. doi:10.1016/j.chom.2012.08.005

12. Chen Y, Chen M, Zhang Y, et al. Broad-spectrum neutralization of pore-forming toxins with human erythrocyte membrane-coated nanosponges. Adv Healthc Mater. 2018;7(13):e1701366. doi:10.10 02/adhm.201701366

13. Nel A, Xia T, Madler L, Li N. Toxic potential of materials at the nanolevel. Science. 2006;311(5761):622-627. doi:10.1126/science. 1114397
14. Hoshino Y, Koide H, Furuya K, et al. The rational design of a synthetic polymer nanoparticle that neutralizes a toxic peptide in vivo. Proc Natl Acad Sci U S A. 2012;109(1):33-38. doi:10.1073/pnas.1112828109

15. Wang F, Gao W, Thamphiwatana S, et al. Hydrogel retaining toxin-absorbing nanosponges for local treatment of methicillin-resistant staphylococcus aureus infection. Adv Mater. 2015;27(22):3437-3443. doi:10.1002/adma.201501071

16. Jiang Y, Krishnan N, Heo J, Fang RH, Zhang L. Nanoparticlehydrogel superstructures for biomedical applications. J Control Release. 2020;324:505-521. doi:10.1016/j.jconrel.2020.05.041

17. Tao J, Xu X, Wang S, et al. Polydiacetylene-nanoparticlefunctionalized microgels for topical bacterial infection treatment. ACS Macro Lett. 2019;8:563-568. doi:10.1021/acsmacrolett.9b00196

18. Shende P, Desai D, Gaud RS. Hybrid nanosponges. In: Trotta F, Mele A, editors. Nanosponges. Weinheim: Wiley-VCH; 2019:173-192.

19. Zhang Y, Gao W, Chen Y, et al. Self-assembled colloidal gel using cell membrane-coated nanosponges as building blocks. ACS Nano. 2017;11(12):11923-11930. doi:10.1021/acsnano.7b06968

20. Gou M, Qu X, Zhu W, et al. Bio-inspired detoxification using 3D-printed hydrogel nanocomposites. Nat Commun. 2014;5:3774. doi: $10.1038 /$ ncomms 4774

21. Gao W, Vecchio D, Li J, et al. Hydrogel containing nanoparticle-stabilized liposomes for topical antimicrobial delivery. ACS Nano. 2014;8(3):2900-2907. doi:10.1021/nn500110a

22. Gao W, Zhang Y, Zhang Q, Zhang L. Nanoparticle-hydrogel: a hybrid biomaterial system for localized drug delivery. Ann Biomed Eng. 2016;44(6):2049-2061. doi:10.1007/s10439-016-1583-9

23. Xiao Y, Gu Y, Qin L, et al. Injectable thermosensitive hydrogel-based drug delivery system for local cancer therapy. Colloids Surf B Biointerfaces. 2021;200:111581. doi:10.1016/j.colsurfb.2021.111581

24. Xue B, Qu Y, Shi K, et al. Advances in the application of injectable thermosensitive hydrogel systems for cancer therapy. $J$ Biomed Nanotechnol. 2020;16(10):1427-1453. doi:10.1166/jbn.2020.2988

25. Lee ALZ, Voo ZX, Chin W, et al. Injectable coacervate hydrogel for delivery of anticancer drug-loaded nanoparticles in vivo. ACS Appl Mater Interfaces. 2018;10(16):13274-13282. doi:10.1021/acsami.7b 14319

26. Qian H, Qian K, Cai J, Yang Y, Zhu L, Liu B. Therapy for gastric cancer with peritoneal metastasis using injectable albumin hydrogel hybridized with paclitaxel-loaded RBC membrane nanoparticles. ACS Biomater Sci Eng. 2019;5(2):1100-1112. doi:10.1021/acsbiomaterials.8b01557

27. Ohta S, Hiramoto S, Amano Y, et al. Intraperitoneal delivery of cisplatin via a hyaluronan-based nanogel/in situ cross-linkable hydrogel hybrid system for peritoneal dissemination of gastric cancer. Mol Pharm. 2017;14(9):3105-3113. doi:10.1021/acs.molpharmaceut.7b00349

28. Constantin M, Bucatariu SM, Doroftei F, Fundueanu G. Smart composite materials based on chitosan microspheres embedded in thermosensitive hydrogel for controlled delivery of drugs. Carbohydr Polym. 2017;157:493-502. doi:10.1016/j.carbpol.2016.10.022

29. Lin HC, Anggelia MR, Cheng CC, et al. A mixed thermosensitive hydrogel system for sustained delivery of tacrolimus for immunosuppressive therapy. Pharmaceutics. 2019;11(8):413. doi:10.3390/ pharmaceutics11080413

30. Jayaramudu T, Varaprasad K, Reddy KK, Pyarasani RD, AkbariFakhrabadi A, Amalraj J. Chitosan-pluronic based $\mathrm{Cu}$ nanocomposite hydrogels for prototype antimicrobial applications. Int $J$ Biol Macromol. 2020;143:825-832. doi:10.1016/j.ijbiomac.2019.09.143

31. Xiaoyu C, Blenda Chi Kwan W, Hubiao C, et al. Long-lasting insulin treatment via a single subcutaneous administration of liposomes in thermoreversible pluronic ${ }^{\circledR}$ f127 based hydrogel. Curr Pharm Des. 2017;23(39):6079-6085.

32. Nie S, Hsiao WL, Pan W, Yang Z. Thermoreversible pluronic f127-based hydrogel containing liposomes for the controlled delivery of paclitaxel: in vitro drug release, cell cytotoxicity, and uptake studies. Int $J$ Nanomedicine. 2011;6:151-166. doi:10.2147/IJN. S15057 
33. Shriky B, Kelly A, Isreb M, et al. Pluronic F127 thermosensitive injectable smart hydrogels for controlled drug delivery system development. J Colloid Interface Sci. 2020;565:119-130. doi:10. 1016/j.jcis.2019.12.096

34. Schwoch GSH, Passow H. Preparation and properties of human erythrocyte ghosts. Mol Cell Biochem. 1973;2(2):197-218. doi:10. 1007/BF01795474
35. Akhtar MF, Hanif M, Ranjha NM. Methods of synthesis of hydrogels ... A review. Mol Cell Biochem. 2016;24(5):554-559.

\section{Publish your work in this journal}

The International Journal of Nanomedicine is an international, peerreviewed journal focusing on the application of nanotechnology in diagnostics, therapeutics, and drug delivery systems throughout the biomedical field. This journal is indexed on PubMed Central, MedLine, CAS, SciSearch ${ }^{\mathbb{R}}$, Current Contents ${ }^{\mathbb{R}} /$ Clinical Medicine, $^{-}$
Journal Citation Reports/Science Edition, EMBase, Scopus and the Elsevier Bibliographic databases. The manuscript management system is completely online and includes a very quick and fair peer-review system, which is all easy to use. Visit http://www.dovepress.com/ testimonials.php to read real quotes from published authors. 